

\title{
Contrast enhancement of Micro Dose X-ray images
}

\author{
Paolo Irrera, Isabelle Bloch, Maurice Delplanque
}

\section{To cite this version:}

Paolo Irrera, Isabelle Bloch, Maurice Delplanque. Contrast enhancement of Micro Dose X-ray images. IEEE International Symposium on Biomedical Imaging - ISBI 14', Apr 2014, Beijing, China. pp.489 - 492, 10.1109/ISBI.2014.6867915 . hal-01096613

\section{HAL Id: hal-01096613 https://hal.science/hal-01096613}

Submitted on 17 Dec 2014

HAL is a multi-disciplinary open access archive for the deposit and dissemination of scientific research documents, whether they are published or not. The documents may come from teaching and research institutions in France or abroad, or from public or private research centers.
L'archive ouverte pluridisciplinaire HAL, est destinée au dépôt et à la diffusion de documents scientifiques de niveau recherche, publiés ou non, émanant des établissements d'enseignement et de recherche français ou étrangers, des laboratoires publics ou privés. 


\title{
CONTRAST ENHANCEMENT OF MICRO DOSE X-RAY IMAGES
}

\author{
Paolo Irrera ${ }^{\star \dagger} \quad$ Isabelle Bloch Maurice Delplanque $^{\dagger}$ \\ *Institut Mines Telecom, Telecom ParisTech, CNRS LTCI, Paris, France - name.surname@ telecom-paristech.fr \\ ${ }^{\dagger}$ EOS imaging, Paris, France - mdelplanque@eos-imaging.com
}

\begin{abstract}
A multi-scale (MS) decomposition method for contrast enhancement of Micro Dose (MD) X-ray images is presented in this paper. First, we get a denoised version of the input exploiting a non-local means filter with adaptable parameters setting that we defined in a former approach. Then, the MS representations of the input and of its denoised version are combined to obtain an optimal image in terms of preservation of details and noise attenuation. The efficiency of the algorithm is demonstrated by quantitative and qualitative assessments on both phantoms and clinical MD images.
\end{abstract}

Index Terms - Micro Dose X-ray imaging, Multi-scale decomposition, Non-local means filter

\section{INTRODUCTION}

Contrast enhancement is often applied on X-ray images because it improves the local contrast, thus easing the diagnosis. In radiology, while the large density variations occur mainly over low frequencies, the most relevant details belong to high frequencies [1]. The linear multi-scale (MS) methods, e.g. the Laplacian Pyramid [2], allow separating an image in scales of spatial frequency. Thus, they can be used to enhance X-ray images [1], [3]. However, they raise two issues. The first problem is the creation of halo artifacts due to smoothing of strong edges. In other image processing domains (e.g. digital photography), edge-preserving algorithms [4] address this question. In radiology, these artifacts constitute an actual problem in presence of radio-opaque objects, e.g. prosthesis. Indeed, shadows may appear around the prosthesis making difficult for the radiologist to evaluate the health of the surrounding tissues. Excluding this case, the halo artifacts are very limited and can, thus, be tolerated [3]. This is an assumption made in this paper. The second problem concerns the analysis of noisy images. The noise is represented at fine levels of the decomposition and, so, mixed up with details relevant for the diagnosis. Thus, the challenge consists in enhancing the fine details while avoiding boosting the noise. A common solution is the noise containment [1]: the noisy coefficients are not enhanced, but not attenuated either, which guarantees to preserve all the information.

In this project we process Micro Dose (MD) images acquired with the X-ray system EOS. This system is based on Charpak's high energy particle detector [5]. Frontal and lateral images of the full body of the patient can be acquired in upright position. EOS has been shown to provide spine radiographs at 6 to 9 times less dose than computer radiography [6], which is why we call Low Dose (LD) the normally exposed EOS images. Micro Dose is a new EOS feature that allows a further dose reduction equal to 5 to 7 times w.r.t. LD acquisitions. The amount of dose delivered to the patient during a MD

The PhD work of P. Irrera is supported by a grant from ANRT. exam is comparable to 5 to 10 days of natural exposure. This feature is clearly of great interest given the increasing concerns on patients health due to over-exposed acquisitions [7]. These images are mainly useful for follow-up exams (e.g. measure of scoliosis angle trending). Nevertheless, the reduction of the flux of photons implies a rise of the amount of noise. So, since the noise propagates through coarser levels than the first one, the aforementioned noise containment technique is not optimal anymore. Then, a possible method consists in denoising the image before enhancing the local contrast [8], [9]. Nevertheless, this kind of approach could lead to an artificial look of structures at medium frequencies, e.g. the edges of the vertebrae. So, at present, MD images are processed in clinical routine with the same family of algorithms used on LD images.

Our contribution consists in optimizing the visualization of MD images in terms of: noise reduction, contrast enhancement and preservation of their natural aspect. We define a new MS decomposition that is not biased by the presence of noise that affects the input image. For this aim, we combine the decomposition of the original image and of its denoised version, including the denoising directly into the enhancement loop. The denoised image is obtained using a Non-Local means (NL-means) filter [10], [11] with an adaptable parameter control related to X-ray absorption that we described in [9]. We want to stress that the proposed method is not just a MS denoising technique. Indeed, since we aim at obtaining a well contrasted output image, the reduction of the noise represents only half of the whole matter.

The method is explained in Section 2. In Section 3 we validate our algorithm by testing it on phantoms and MD images. Conclusions and future developments are outlined in Section 4.

\section{CONTRAST ENHANCEMENT OF NOISY IMAGES}

The algorithm is divided into three main steps (see Fig. 1) : the adaptive NL-means filter (block 1), the MS analysis (block 2) and the MS synthesis (block 3 ).

\subsection{Adaptive NL-means denoising}

The NL-means filter denoises an image through a representation in sub-regions called patches. The interest of working with patches lies in the robustness to noise and good representation of texture [11]. Formally, given a noisy image $I$, the denoised pixel at the spatial location $x_{i}$ is estimated as a weighted sum:

$$
\hat{I}\left(x_{i}\right)=\frac{\sum_{j=1}^{|\Omega|} \varsigma_{i, j} I\left(x_{j}\right)}{\sum_{j=1}^{|\Omega|} \varsigma_{i, j}}, \quad \varsigma_{i, j}=\exp \left(-\frac{d\left(P_{i}, P_{j}\right)}{\alpha}\right)
$$

where $\Omega$ is the size of a window centered at pixel $x_{i}$ that determines which pixels have to be averaged. The weights $\varsigma_{i, j}$ are defined in 


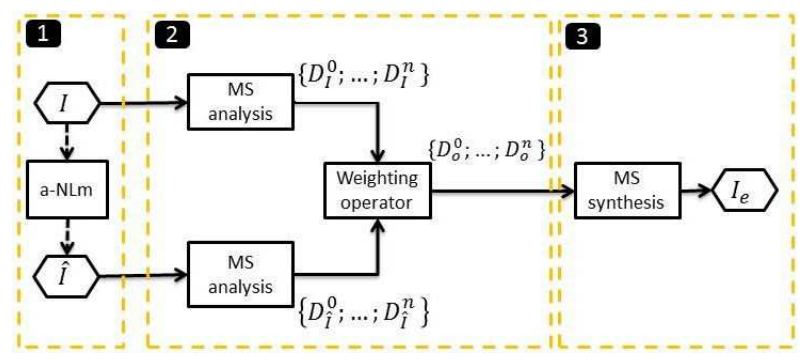

Fig. 1: Architecture of the proposed method. $I=$ input image; $\hat{I}=$ denoised image; $D_{I}^{i}=$ detail image at level $i$ from the decomposition of the image $I$.

function of the similarity between the patches $P_{i}$ and $P_{j}$ centered respectively at the pixels $x_{i}$ and $x_{j}$. The distance function $d($.$) is defined$ according to the type of noise that corrupts the image. It is generally the Euclidean distance, but other functions can be defined when the noise is not Gaussian [11]. Finally, $\alpha$ is the bandwidth of the exponential kernel.

In a former work [9], we pointed out that the choice of one value of $\alpha$ on a full body $\mathrm{X}$-ray image is sub-optimal: there is a strong risk of loosing anatomical information in low absorption regions, e.g. bone textures. Thus, considering that the noise is stronger in low incident $\mathrm{X}$-ray dose areas [1], we defined a map of different values empirically related to the thickness of the body of the patient. In this work we exploit the same idea. However the definition of the alpha map is simplified. First, the envelop of the body is segmented by thresholding the image in the linear domain ${ }^{1}$. Then, using the cumulative histogram of gray levels of the previously segmented image, we define a given number of regions of increasing thickness. Subsequently, we compute the local standard deviation image and label each region with the most frequent value in it, obtaining the noise level image. Finally, the alpha map is proportional to this noise level image.

Even if the noise model could be described as Poisson/Gaussian mixture, we employ an Euclidean distance to compare the patches. The signal dependent nature of the noise is taken into account through the alpha map that changes in function of the thickness. Thus, the estimation of the noise free image is practically unbiased.

\subsection{Analysis of noise corrupted images}

The analysis step is the core of the algorithm. The term analysis indicates the decomposition in scale levels $S_{I}^{0}, S_{I}^{1}, \ldots, S_{I}^{n}$ and detail levels $D_{I}^{0}, D_{I}^{1}, \ldots, D_{I}^{n-1}$. The scale level $S_{I}^{i}$ is the smoothed version of scale $S_{I}^{i-1}$ (with $S_{I}^{0}=I$ ), i.e. $S_{I}^{i}=f_{i}\left(S_{I}^{i-1}\right)$ where $f_{i}($.$) is a Gaussian-like$ filter. We exploit an undecimated decomposition to avoid aliasing artifacts due to non-linear processing in the reconstruction. We use a simpler decomposition technique than the wavelets because this last one has been proven to be inappropriate for the contrast enhancement of medical images [3]. The detail level is the output of a band-pass filter: $D_{I}^{i-1}=S_{I}^{i-1}-S_{I}^{i}$.

We define the optimal decomposition using the levels obtained from the original image $I$ and its denoised version $\hat{I}$. Precisely, the output detail level $D_{O}^{i}$ is the weighted mean of the noisy detail level $D_{I}^{i}$ and the denoised detail level $D_{\hat{I}}^{i}$. The decomposition of $\hat{I}$ has the advantage of representing only details proper to the signal, avoiding

\footnotetext{
${ }^{1}$ The image in the linear domain is obtained after the calibration that is the first step of the image processing reconstruction chain. Then, considering that the X-rays follow an exponential law, the image is converted to the logarithmic domain and we obtain $I$.
}

the propagation of noise at levels $i>0$. Nevertheless, the attenuation of coefficients can entail an excessive regularization of the outcome. However, thanks to the adaptive NL-means filter, we can preserve the information in low absorption regions and the edges are not smoothed. Then, we can detect these spots of the image and use the coefficients from the noisy decomposition to represent them. On the contrary, the flat regions are represented by the coefficients from the denoised image. This idea is formalized by the following weighting function:

$$
w_{i}=\left(\frac{\left|\nabla\left(S_{I}{ }^{i}\right)\right|}{\left|\nabla\left(S_{I}{ }^{i}\right)\right|+\left|\nabla\left(S_{\hat{I}}^{i}\right)\right|}\right)^{\beta} \quad, \beta \geqslant 1
$$

where $|\nabla(I)|$ is the gradient magnitude of an image $I$ and $\beta$ is a userdefined parameter. The value of $w_{i}$ is low when the gradient of the scale image obtained from the denoised image is higher than the gradient of the scale image obtained from the noisy image. This happens when an edge is preserved and the noise around it is reduced. In this case, we have detected an edge and, so, we give a higher weight to the coefficients from the noisy image. Conversely, high values of $w_{i}($.$) imply a reduction of noise in smooth regions and the denoised$ coefficients are retained. The parameter $\beta$ controls the power of the denoising: the higher it is, the lower are the weights. For example, assuming that the gradients are equivalent in a location $x$, if $\beta=1$ then the coefficients have the same importance $(w(x)=0.5)$, instead if $\beta=2$ the noisy coefficients are more relevant $(w(x)=0.25)$. Note that the values of $w_{i}($.$) are lower and lower at coarser scales because$ the power of the noise gets less and less important. Finally, the output levels are obtained as $D_{O}^{i}=w_{i} D_{\hat{I}}^{i}+\left(1-w_{i}\right) D_{I}^{i}$, resulting in a denoised image but preserving the coefficients from strong edges.

\subsection{Synthesis of noise corrupted images}

The synthesis is the process that rebuilds the image from the detail levels and the low frequency (LF) residuals obtained during the analysis step. The enhanced image is obtained by summation:

$$
I_{e}=\sum_{i=0}^{n-1} b_{i}\left(D_{O}^{i}\right)+\operatorname{lut}\left(S_{\hat{I}}^{n}\right)
$$

The details $D_{O}^{i}$ are boosted by the non linear function $b_{i}$. In this paper, we use the boosting function defined by Stahl et al. [1]:

$$
b_{i}(d)= \begin{cases}G_{i}\left(1-\frac{|d|}{d_{0}}\right)^{p_{i}}+1 & \text { if }|d| \leqslant d_{0} \\ 1 & \text { if }|d|>d_{0}\end{cases}
$$

where $G_{i}$ is the maximum gain, $p_{i}$ controls how fast the amplification decreases toward 1 and $d_{0}$ is the amplitude value after which the coefficients are not enhanced. The value of $d_{0}$ is defined according to the binary levels used to encode the image. Instead, $G_{i}$ and $p_{i}$ are defined according to the scanned anatomical region and the application [1]. To treat the LF residual, we apply a sigmoid-like function (lut) that attenuates the low values of the LF residual and preserves the strong ones. We define $\gamma \in[0,1)$ as the strongest attenuation. The point where the function changes of convexity is fixed from the intersection of the cumulative histogram of the LF residual with the cumulative histogram of an ideal uniform distribution. On an X-ray image this allows maximizing the dynamic of the image.

\section{VALIDATION OF THE METHOD}

The proposed method was validated on phantoms and MD clinical images encoded on 16 bits. The images of the phantom portrait all 
the same object (standard PHD5000 fluoro phantom) but with different acquisition parameters that are: the peak kilo voltage output of the X-ray generator $(\mathrm{kV})$, the $\mathrm{X}$-ray tube current in $\mathrm{mA}(\mathrm{mA})$ and the speed factor of the $\mathrm{C}$-scan $(\mathrm{C} \in\{1,2, \ldots, 8\}$, the higher it is the lower is the speed). The phantoms are used to quantify the improvement in terms of signal to noise ratio (SNR), contrast to noise ratio (CNR) and dynamic (DYN) that represents the global contrast. Our clinical data set includes 13 Micro Dose studies of spine acquired at the hospital Robert Debré, Paris ${ }^{2}$. The minimum and maximum values of dose (Air Kerma) are 7.37 $\mu G y$ and $29.44 \mu G y$ and $17.43 \mu G y$ and $55.13 \mu G y$ for, respectively, frontal and lateral acquisitions.

The parameters used in the algorithm are mostly defined in function of the content of the image. Indeed, the alpha map is proportional to the noise level map (see Sec 2.1); for all the results reported here the proportionality factor is equal to 4 . The patches and the windows of the NL-means filter are respectively of size $7 \times 7$ and $21 \times 21$. In the analysis the only parameter to be defined is $\beta$ (see Sec. 2.2). This parameter allows weighting the contribution of the denoising and it is the only one that needs to be changed as a function of dose. Thus, for our tests we used $\beta=1.5$ and $\beta=3$ respectively on MD and LD data. Finally, the synthesis parameters are practically defined on the basis of the type of study [1]. For all our tests, even those on phantoms, we opted for parameters that aim at enhancing the details of the spine. Thus, the high/medium frequency detail levels $(i \in[1,3])$ are the most enhanced. Besides, we set $\gamma=0.5$ to process the LF residual.

The method was compared with two noise containment based algorithms that are usually used by EOS in clinical routine. One is employed on LD images and the other on MD images; they will be respectively named hereafter EOS-LD and EOS-MD. This last one is specifically designed to enhance the spine, which is why we chose this category of study.

\subsection{Results on phantoms}

The SNR is given by the ratio of the mean signal on the standard deviation in a constant region. The CNR is the ratio of the difference of signal between the first disk from the left in Fig. 2 and the background, on the standard deviation of this last one. Then, DYN is the difference of the average signal of the white and black tablets (see the upper zone of the image in Fig. 2). The results obtained with the proposed approach are better for all the metrics both in the LD and MD cases as shown, respectively, in Tab. 1 and 2. In details, with

\begin{tabular}{|c|c|c|c|c|c|c|c|c|c|}
\hline \multicolumn{4}{|c|}{ Params. } & \multicolumn{3}{c|}{ EOS-LD } & \multicolumn{3}{c|}{ Prop. } \\
\hline $\mathrm{kV}$ & $\mathrm{mA}$ & $\mathrm{C}$ & Dose $\mu \mathrm{Gy}$ & SNR & CNR & DYN & SNR & CNR & DYN \\
\hline 70 & 200 & 4 & 79.36 & 18.81 & 0.92 & $55 \%$ & 35.05 & 3.70 & $61 \%$ \\
\hline 90 & 200 & 4 & 124.04 & 26.92 & 1.57 & $51 \%$ & 49.73 & 5.82 & $60 \%$ \\
\hline 120 & 200 & 4 & 197.94 & 39.82 & 2.12 & $53 \%$ & 67.21 & 7.30 & $61 \%$ \\
\hline
\end{tabular}

Table 1: Quantitative assessment on phantoms: Low Dose

\begin{tabular}{|c|c|c|c|c|c|c|c|c|c|}
\hline \multicolumn{4}{|c|}{ Params. } & \multicolumn{3}{|c|}{ EOS-MD } & \multicolumn{3}{|c|}{ Prop. } \\
\hline kV & mA & C & Dose $\mu G y$ & SNR & CNR & DYN & SNR & CNR & DYN \\
\hline 60 & 80 & 3 & 10.29 & 12.93 & 2.2 & $62 \%$ & 16.22 & 3.51 & $70 \%$ \\
\hline 65 & 80 & 4 & 16.97 & 15.75 & 1.37 & $56 \%$ & 22.31 & 2.58 & $63 \%$ \\
\hline 72 & 125 & 4 & 34.08 & 19.29 & 2.30 & $58 \%$ & 26.99 & 3.80 & $64 \%$ \\
\hline
\end{tabular}

Table 2: Quantitative assessment on phantoms: Micro Dose

\footnotetext{
${ }^{2}$ We would like to thank the Professor G. Sebag and the Doctor M. Alison from the hospital Robert Debré for providing the anonymized RAW images and agreeing to use them for our research purposes
}

LD images, the SNR, the CNR and the DYN are improved on average by, respectively, $\times 1.80, \times 3.72$ and 8 points. Besides, the details are totally preserved as observed in Fig. 2, e.g. see the high contrast resolution grid and the writing. These results suggest that even in some cases with a sufficient amount of dose, the methods based on noise containment can fail in avoiding the noise boosting. Instead, the method that we propose proves to be more robust. As for the MD



(a)

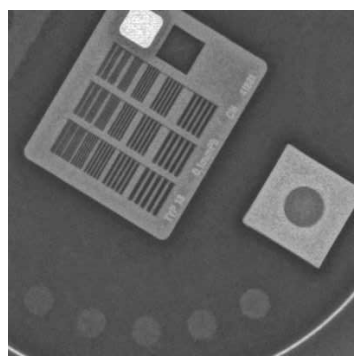

(b)
Fig. 2: Crop of the phantom 70kV and 200mA: (a) EOS-nD, (b) Proposed

case, the SNR, the CNR and the DYN are improved on average by, respectively, $\times 1.36, \times 1.71$ and 7 points. The improvement in terms of SNR and CNR is still significant even if it is less important w.r.t. the statistics obtained in the LD case because the noise is stronger. However, the scores of the DYN reveal a much better global contrast offered by our solution. Finally, the spatial resolution is not reduced in this case either.

\subsection{Results on Micro Dose spine images}

The validation of the method on clinical images was done first by a subjective comparison between the three enhancement algorithms. In details, we study the lumbar region cropped from one of the MD images composing our clinical data set. The image enhanced with EOS-LD (Fig. 3(b)) has not an ideal global contrast. For example, the spine and the surrounding soft tissues have nearly the same gray level. Besides, the presence of noise is evident. This probably implies that the medium frequency details are not sufficiently enhanced and that the noise containment is not efficient. The solution offered by EOS-MD (Fig. 3(c)) is better, which justifies the current choice of this algorithm in the MD context. However, we can clearly see background noise in smooth regions. This is due to the inability of the MS decomposition to confine the noise at the finest level. Our algorithm (Fig. 3(d)) allows smoothing the most regular regions by reducing the visibility of the noise, while preserving the shapes of the vertebrae, i.e. only the relevant information of the image is enhanced. Finally, the global contrast is well balanced.

We used Average Local Variance (ALV) metrics as objective assessment [12]. This approach consists in, first, classifying each pixel in three categories of regions, i.e. smooth, details and edges. Then, the average standard deviation is computed in each region obtaining, respectively, ALVS, ALVD and ALVE. Generally, the best contrast enhancement algorithm has low values of ALVS and ALVE, which quantify respectively the noise enhancement and the presence of halos, and high ALVD, which quantifies the details boosting. For every image of the clinical data set, we defined the three regions by a basic threshold classification of the detail levels $D_{\hat{I}}^{i}$ and we computed the scores on the input image and on the outputs of the compared contrast enhancement algorithms. The averages of the metrics obtained on the whole data set are reported in Tab. 3. The algorithms 


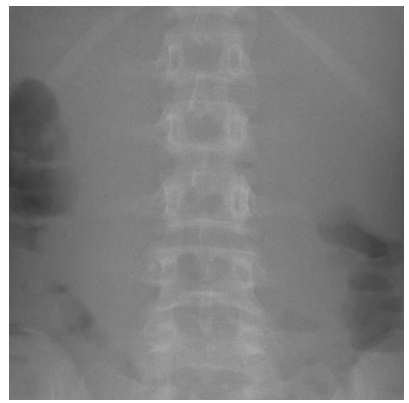

(a)

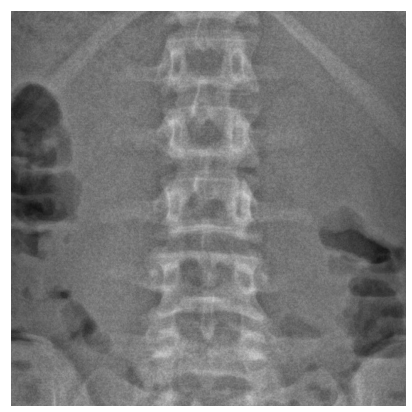

(c)

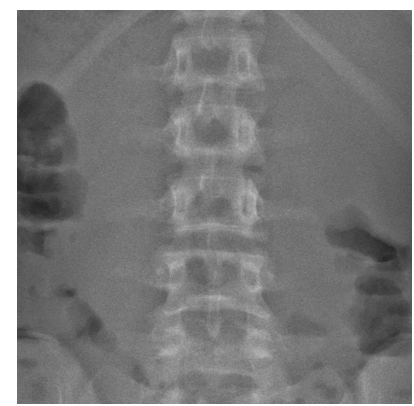

(b)

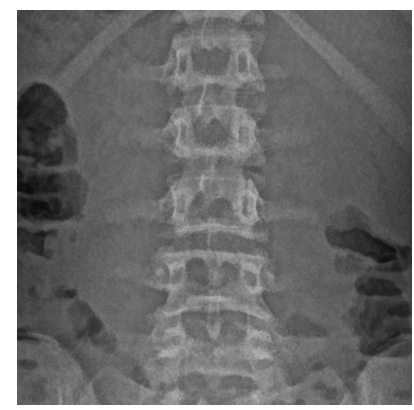

(d)
Fig. 3: Crop from a spine image; dose $=14.64 \mu G y$ : (a) Low contrast and noisy image, (b) EOS-LD, (c) EOS-MD, (d) Our method.

EOS-LD and EOS-MD have similar scores: the significant enhancement of the details coincides with an even bigger enhancement in smooth regions. Indeed, with EOS-MD, ALVS and ALVD are increased w.r.t the scores of the input image by respectively $\times 2.73$ and $\times 2.51$. The proposed approach has lower ALVD compared to the other two methods. However, the smooth regions are much less enhanced too. Indeed, ALVS and ALVD are increased w.r.t the scores of the input image by respectively $\times 2.03$ and $\times 2.18$. Thus, thanks to the proposed algorithm we are able to enhance more the details than the noise. Finally, we did not notice halo artifacts on any outcomes of the three compared algorithms. However, the lowest ALVE value was obtained with the proposed method, which suggests that it may be less prone to this issue too.

\begin{tabular}{|c|c|c|c|}
\hline & ALVS & ALVD & ALVE \\
\hline Input & 442.98 & 561.93 & 848.83 \\
\hline EOS-LD & 1175.18 & 1375.49 & 1792.57 \\
\hline EOS-MD & 1210.09 & 1411.26 & 1756.96 \\
\hline Prop. & 899.55 & 1227.70 & 1657.70 \\
\hline
\end{tabular}

Table 3: Average ALV scores on the clinical data set

\section{CONCLUSIONS}

In this paper we proposed an original method to decompose and enhance X-ray MD images that exploits the information provided by our adaptive NL means filter directly in the MS decomposition loop. An optimal representation of the details levels is obtained to limit the enhancement to relevant information of the image while avoiding too regular outcomes. The efficiency of the algorithm is demonstrated by quantitative and qualitative assessments on both phantoms and clini- cal MD images. As shown by the scores obtained on the phantoms, it seems possible to extend the method to LD images too. Thus, we intent to fulfill our study with tests on clinical LD images. Besides, we want to quantify how much we can decrease the amount of dose and still be able, thanks to the proposed method, to obtain a diagnostic image that targets other applications than follow-up exams. In this perspective, we are going to involve radiologists in the study to get a quantitative clinical assessment of the image quality.

\section{REFERENCES}

[1] M. Stahl, T. Aach, S. Dippel, T. Buzug, R. Wiemker, and U. Neitzel, "Noise-Resistant Weak-Structure Enhancement for Digital Radiography," in SPIE Vol. 3661: Medical Imaging 99: Image Processing, K. M. Hanson, Ed., San Diego, USA, February 1999, pp. 1406-1417.

[2] P.J. Burt and E.H. Adeldon, "The Laplacian Pyramid as a Compact Image Code," IEEE Transactions on Communications, vol. 31, no. 4, pp. 532-540, 1983.

[3] S. Dippel, M. Stahl, R. Wiemker, and T. Blaffert, "Multiscale Contrast Enhancement for Radiographies: Laplacian Pyramid Versus Fast Wavelet Transform," IEEE Transactions on Medical Imaging, vol. 21, no. 4, pp. 343 -353, April 2002.

[4] S. Paris, S.W. Hasinoff, and J. Kautz, "Local Laplacian Filters: Edge-aware Image Processing with a Laplacian Pyramid," ACM Transaction on Graphics, vol. 30, no. 4, pp. 68:1 - 68:12, July 2011.

[5] J. Dubousset, G. Charpak, and W. Skalli, "Skeletal and spinal imaging with EOS system," Archives de Pédiatrie, vol. 15, no. 5, pp. 665-666, 2008.

[6] S. Deschênes, G. Charron, and G. Beaudoin, "Diagnostic imaging of spinal deformities: reducing patients radiation dose with a new slot-scanning X-ray imager," The Spine Journal, vol. 35, no. 9, pp. 989-994, 2010.

[7] R. Smith-Blindman, D.L. Miglioretti, and E. Johnson, "Use of Diagnostic Imaging Studies and Associated Radiation Exposure for Patients Enrolled in Large Integrated Health Care Systems, 1996-2010," The Journal of American Medical Association, vol. 307, no. 22, pp. 2400-2409, June 2012.

[8] M. Sakata and K. Ogawa, "Noise Reduction and Contrast Enhancement for Small-Dose X-ray Images in Wavelet Domain," in IEEE Nuclear Science Symposium Conference Record, October 2009, pp. $2924-2929$.

[9] P. Irrera, I. Bloch, and M. Delplanque, "A Denoising Method for Whole-Body Low-Dose X-Ray Images with Adaptable Parameter Control," in International Symposium on Biomedical Imaging: From Nano to Macro, April 2013, pp. 1228-1231.

[10] A. Buades, B. Coll, and J-M. Morel, "A Non-Local Algorithm for Image Denoising," in IEEE Conference on Computer Vision and Pattern Recognition, 2005, vol. 2, pp. 60-65.

[11] C.-A. Deledalle, L. Denis, and F. Tupin, "How to compare noisy patches? Patch similarity beyond Gaussian noise," International Journal of Computer Vision, vol. 99, no. 1, pp. 86-102, 2012.

[12] D-C. Chang and W-R. Wu, "Image Contrast Enhancement Based on a Histogram Transformation of Local Standard Deviation," IEEE Transactions on Medical Imaging, vol. 17, no. 4, pp. 518 -531, August 1998. 